eradication rates. However, these regimens have the drawbacks of severe side effects, high cost, and low compliance due to the use of multiple antibiotic agents. Furthermore, usage of multiple antibiotic agents in $H$. pylori treatment have some concern to increase future its antibiotic resistance. Thus, novel regimens that enable minimum antibiotic use, prevent future antibiotic resistance, and achieve sufficient eradication rates, are required. Vonoprazan and amoxicillin dual therapy could be an alternative treatment regimen for $H$. pylori eradication. This study aimed to investigate the efficacy of vonoprazanbased dual therapy as first-line $H$. pylori treatment compared with vonoprazan-based triple therapy.

Methods This study will enroll 320 patients with $H$. pylori infection, confirmed by culture test, at seven institutions in Japan. The enrolled patients will be randomly assigned to either VA-dual therapy (vonoprazan $20 \mathrm{mg}+$ amoxicillin 750 mg twice/day for 7 days) or VAC-triple therapy (vonoprazan $20 \mathrm{mg}+$ amoxicillin $750 \mathrm{mg}$ + clarithromycin $200 \mathrm{mg}$ twice/ day for 7 days) at a 1:1 allocation ratio with stratification by age, sex, amoxicillin resistance of $H$. pylori, clarithromycin resistance of $H$. pylori, and institution. The primary outcome of this study is $H$. pylori eradication rates in both groups. Eradication success is evaluated at least 4 weeks after the treatment period using the ${ }^{13} \mathrm{C}$-urea breath test. Comparative non-inferiority of the two groups will be assessed through a derivation of a two-sided 95\% confidence interval (CI) and hypothesis testing. The secondary endpoints are adverse events rates.

Results This study was approved by the Institutional Review Board of Nihon University School Hospital on September 18, 2018. The recruitment started from October 2018 and will continue for an 8-month period.

Conclusions The findings of this study will be submitted to a peer-reviewed journal. Abstracts will be submitted to relevant national and international conferences. This study is registered with the University Hospital Medical Information Network (UMIN) Clinical Trials Registry (www.umin.ac.jp/ctr/; identification No.: UMIN00003414).

\section{IDDF2019-ABS-0146 EFFICACIES OF TAILORED THERAPY VERSUS GUIDELINE-RECOMMENDED EMPIRICALTHERAPIES FOR ERADICATION OF HELICOBACTER PYLORI - A TREND SURVEY OVER 20 YEARS IN TAIWAN (1999-2018)}

${ }^{1}$ Chien-Chih Tung*, Chi-Tan Hu, ${ }^{3}$ Chun-Jung Lin, ${ }^{4}$-Nan Kuo, ${ }^{1}$ Bor-Ru Lin, ${ }^{5}$ HongLong Wang, ${ }^{6}$ Jin-De Chen, ${ }^{7}$ Mu-Liang Cheng, ${ }^{1}$ Chia-Tung Shun, ${ }^{3}$ Huei-Mi Li, ${ }^{2}$ JuiSheng Hung, ${ }^{2}$ Wei-Yi Lei, ${ }^{1}$ Ming-Jium Shieh, ${ }^{1}$ Jau-Min Wong, ${ }^{8}$ John Yung-Chong Kao. ${ }^{1}$ National Taiwan University Hospital, Taiwan; ${ }^{2}$ Buddhist Tzu Chi General Hospital, Taiwan; ${ }^{3}$ School of Pharmacy, National Taiwan University, Taiwan; ${ }^{4}$ National Taiwan University Hospital Hsin-Chu Branch, Taiwan; ${ }^{5}$ Department of Statistics, National Taipei University, Taiwan; ${ }^{6}$ National Taiwan University Hospital Bei-Hu Branch, Taiwan; ${ }^{7}$ Mennonite Christian Hospital, Taiwan; ${ }^{8}$ University of Michigan, USA

\subsection{6/gutjnl-2019-IDDFabstracts. 166}

Background The empiric therapies for $H$. pylori infection in clinical guidelines have been widely used. However, the cure rate is decreasing due to the increase of antibiotic resistance. The importance of antimicrobial susceptibility test (AST) has been documented in many consensuses. However, the effect of tailored therapy remains controversial because the AST is rarely offered in most areas. We compared the evolution of treatment efficacy among tailored therapy and some recommended empiric therapies through a trend survey from 1999 to 2018.

Methods This retrospective survey was performed at 2 medical centers and 3 community hospitals in Taiwan. A total of 16,370 treatment naive or failure patients were recruited. Successful $H$. pylori eradication was defined as a negative ${ }^{13} \mathrm{C}$ UBT. The empiric first-line regimens include tailored therapy, clarithromycin-containing triple therapy (CLA-TT), sequential therapy (ST), bismuth-containing quadruple therapy (BQT), and high-dose dual therapy (HDDT). The empiric rescue regimens include tailored therapy, levofloxacin-containing triple therapy (LEV-TT), BQT, and HDDT. We divided the 20 years of follow-up time into 4-year periods for evaluating the trend of treatment efficacies. The E-test was performed to evaluate H. pylori resistance. For the tailored therapy, CLA-TT, LEVTT, BQT, or HDDT was chosen according to the resistance pattern of each patient.

Results The efficacies of tailored therapy, BQT, and HDDT maintain a stable and high efficacy in both first-line and rescue treatment during the study period. However, the efficacies of CLA-TT, ST, and LEV-TT are decreasing year by year. The eradication rate of tailored therapy is significantly higher than that of CLA-TT, ST, LEV-TT, and BQT in recent 4-year period. However, there is no significant difference between the efficacy of tailored therapy and HDDT. The prevalence of $H$. pylori resistance to CLA and LEV increased gradually. In contrast, the resistance rate to amoxicillin and tetracycline remained low.

Conclusions Over the past 20 years, we found that the efficacy of tailored therapy remains relatively stable. Of the recommended empiric therapies, HDDT and BQT have stable therapeutic efficacies and are a good choice of empiric treatment currently.

\section{IDDF2019-ABS-0151 CLINICAL UTILITY OF ENDOSCOPIC RETROGRADE APPENDIX STENTING IN THE OLDEST-OLD PATIENTS}

Jia-Chuan Wu*, Biao Liang, Xiao-Qiao Yang, Rui-Yan Chen, Xiao-Dong Chen, Li-Fang Ye. Guangdong Second Provincial General Hospital, China

\subsection{6/gutjnl-2019-IDDFabstracts.167}

Background To evaluate the indications, efficacy, safety and recurrence rate of endoscopic retrograde appendix stenting for the oldest-old patients aged 80 and over.

Methods Data of the oldest-old patients who went through endoscopic retrograde appendix stenting in our center between July 1st, 2017 and June 30th, 2018 were retrospectively analyzed. The endoscopic procedures included $\underline{4}$ steps: (1) colonoscopic appendiceal intubation, (2) retrograde appendicography, (3) stent drainage and appendiceal decompression, (4) stent extraction $\underline{2}$ weeks later. Main outcome measurements included successful colonoscopic intubation rates, the time to symptom relief, the time to abdominal sign disappearance, increased white blood cell count, procedurerelated complications, and recurrence.

Results 6 patients were enrolled in our study. No immediate endoscopy-related complications and death occurred. All colonoscopic appendiceal intubations were successful. The pain of oldest-old patients was relieved immediately after endoscopic procedures. Abdominal signs, such as tenderness and rebound 